\title{
Planar thermocapillary migration of two bubbles in microgravity environment
}

\author{
R. Sun ${ }^{\text {a) }}$ \\ National Microgravity Laboratory/CAS, Institute of Mechanics, Chinese Academy of Sciences, \\ Beijing 100080, China \\ and Department of Engineering Mechanics, Shanghai Jiao Tong University, Shanghai 200240, China \\ W. R. Hu \\ National Microgravity Laboratory/CAS, Institute of Mechanics, Chinese Academy of Sciences, \\ Beijing 100080, China
}

(Received 3 February 2003; accepted 17 July 2003; published 4 September 2003)

\begin{abstract}
A theoretical investigation is performed on the thermocapillary motion of two bubbles in arbitrary configuration in microgravity environment under the assumption that the surface tension is high enough to keep the bubbles spherical. The two bubbles are driven by the surface tension gradient due to temperature nonuniformity on the surfaces. The bubble interaction is considered for the limit of small Marangoni and Reynolds numbers in the present paper. In order to solve the problem analytically, the method of successive reflections is employed, and then accurate migration velocities of two arbitrarily oriented bubbles in the planar thermocapillary motions are derived. The results demonstrate that two equal-size bubbles exert no influence on the thermocapillary migration of each other at any separation because of the thorough cancellation of the thermal and fluid mechanical interaction effects, and the effect of the large bubble on the motion of the smaller one becomes significant with the two bubbles approaching each other, while the effect of the smaller one on the large remains weak. Moreover, three typical kinds of trajectories of the smaller bubble are identified. (C) 2003 American Institute of Physics. [DOI: 10.1063/1.1607326]
\end{abstract}

\section{INTRODUCTION}

In general, a bubble will move toward the hotter region of a surrounded quiescent liquid subjected to a thermal gradient in microgravity environment. This motion is induced by the thermocapillary effect due to the variation of surface tension with temperature. The phenomenon is of practical importance in materials processing in space applications and also involved in the heat and mass transfer mechanism induced by the bubble migration in microgravity environment.

The thermocapillary motion of gas bubbles was first investigated by Young et al. ${ }^{1}$ They derived a theoretical solution for the migration velocity of an isolated bubble in a bulk liquid with an applied thermal gradient. In microgravity environment, the bubble speed is

$$
u_{\mathrm{YGB}}=\frac{\gamma^{\prime} R\left|\nabla T_{\infty}\right|}{2 \mu} .
$$

Here, $R$ is the radius of the bubble, $\nabla T_{\infty}$ the temperature gradient imposed in the fluid, $\mu$ the dynamic viscosity of the continuous phase, and $\gamma^{\prime}$ refers to the magnitude of variation of the surface tension with the local temperature. The recent literature documents an increasing interest in the understanding of the bubble migration with the advent of space flight programs. Comprehensive reviews have been given by Wozniak et al. $^{2}$ and Subramanian. ${ }^{3}$

a) Author to whom correspondence should be addressed. Electronic mail: drrsun@sjtu.edu.cn
In practical applications of thermocapillary migration, a group of bubbles is usually encountered, and the interaction between them should be more important. In the previous works, Meyyappan et al. ${ }^{4}$ investigated the axisymmetric thermocapillary migration of two bubbles in the quasistatic state by using the bispherical coordinate system. It reveals numerically that two equal-sized bubbles move with the same velocity as if they were isolated. This remarkable result is due to the exact cancellation of the thermal and fluid mechanical two-body interactions. And later, Feuillebois ${ }^{5}$ presented a theoretical confirmation of this numerical conclusion. Although the use of bipolar coordinates is convenient for the axisymmetric case, it has no particular advantage for arbitrary configurations. In subsequent research, Meyyappan and Subramanian ${ }^{6}$ calculated the thermocapillary migration of two bubbles at arbitrary angle to the applied temperature gradient by using a zeroth-order reflection approximation. ${ }^{7}$ Shortly after, Anderson ${ }^{8}$ extended the reflection solution to the first order, and derived the approximate velocities of two arbitrarily oriented droplets valid up to terms of $O\left(r_{12}^{-6}\right)$, where $r_{12}$ is the center-to-center distance between the two droplets. But, both of these approximate expressions mentioned above become invalid with the bubbles approaching each other. Acrivos et al. ${ }^{9}$ predicted the average thermocapillary migration velocity of a cloud of identical bubbles. Keh and Chen ${ }^{10}$ and Wei and Subramanian ${ }^{11}$ employed the boundary-collocation method to study the thermocapillary motion of a small number of bubbles. In their researches, Satrape $^{12}$ and Wang et al. ${ }^{13}$ used twin multipole expansions 
to study the thermocapillary migration of two bubbles oriented arbitrarily with respect to the applied thermal gradient. In this method they first expressed solutions to the governing equations in the form of infinite series with undetermined coefficients, then truncated them to some terms of $O\left(r_{12}^{-n}\right)$, and solved numerically all the coefficients simultaneously. This method, however, becomes extremely cumbersome beyond the first three terms and requires a lot of tedious algebra to generate the matching relations. ${ }^{12}$

Sun and $\mathrm{Hu}^{14}$ have theoretically investigated the interaction between two bubbles aligned parallel to the applied thermal gradient by using the method of successive reflections. The present work extends the previous theoretical research to the case of thermocapillary migrations of two bubbles oriented arbitrarily to a thermal gradient with the aid of the successive reflection method. The crux of using the method lies in understanding the transformations of harmonics and biharmonics between two coordinate systems. Resorting to the method, we obtain analytic solutions in closed form. Using this solution we can predict bubble trajectories so as to determine whether or not the two bubbles will finally collide.

To make the analysis tractable, we also restrict our attention only to the limit of small Reynolds and Marangoni numbers without considering the convective terms in the momentum equations and the energy equation. Generally speaking, such a migration of the two bubbles is an unsteady one, but in many practical applications, such as the glass processing, the Reynolds number based on the bubble radius and the typical velocity, $u_{0}=\gamma^{\prime} R\left|\nabla T_{\infty}\right| / \mu$, is usually low. ${ }^{12}$ In the scaled momentum equation $\operatorname{Re}[\partial \mathbf{u} / \partial t+\mathbf{u} \cdot \nabla \mathbf{u}]=-\nabla p$ $+\nabla^{2} \mathbf{u}$, as indicated by Meyyappan et al., ${ }^{4}$ if the Reynolds number is low and the variation of the velocity with time due to the bubble migration is not large, the Stokes equation gives a reasonable approximation of the two-bubble migration.

The investigation is organized as follows. First, we state the problem and write the governing equations and boundary conditions. This is followed by an analysis of the thermocapillary migration velocities of two bubbles at any angle to the applied thermal gradient. Fundamental solutions of velocity field and temperature distribution for a two-bubble system relative to various initial disturbances (see the Appendix) are necessary to complete this analysis. And, explicit recurrence formulas of the two-bubble migration velocities are constructed from the fundamental solutions using the superposition theorem for linear equations. These formulas are employed in Sec. IV to determine interactions between the two bubbles and describe their migration behaviors. The recurrence formulas seem less intuitive but suitable for numerical computation - this is an advantage of using them. Finally, we comment on this issue in Sec. V.

\section{FORMULATION OF THE PROBLEM}

Two bubbles of radii $R_{1}$ and $R_{2}$ released in a quiescent ambient fluid of density $\rho$ and viscosity $\mu$ will move under the environment of an imposed thermal gradient $\nabla T_{\infty}$. Our attention is focused on the reduced gravity case. Accordingly, the gravitational effects are neglected. We assume that the bubbles remain spherical and are not deformed since capillary number $\mathrm{Ca}$ is negligibly small in many practical applications. Consequently, the normal stress balance on the bubble surfaces is ignored. Moreover, we also neglect the density, the viscosity, and the thermal conductivity of the gas phase inside the bubbles in comparison with those in the host fluid, and assume all liquid properties are independent of temperature with the exception of surface tension, which decreases linearly with temperature, $\gamma=\gamma_{0}-\gamma^{\prime}\left(T-T_{0}\right)$, where $\gamma_{0}$ is the surface tension at a reference constant temperature $T_{0}$.

Using the characteristic velocity, $u_{0}=\gamma^{\prime} R\left|\nabla T_{\infty}\right| / \mu$, the Reynolds number would be extremely small, for example, $\left(\operatorname{Re}<10^{-2}\right)$ in borax glass melt. ${ }^{15}$ And, Marangoni number in the same velocity scale becomes

$$
\mathrm{Ma}=\frac{u_{0} R}{\alpha_{t}}=\frac{\gamma^{\prime} T_{g} R^{2}}{\mu \alpha_{t}},
$$

where $\alpha_{t}$ is the thermal diffusivity and $T_{g}$ denotes $\left|\nabla T_{\infty}\right|$. For borax glass melt, its typical properties are $\rho=2.4 \mathrm{~g} / \mathrm{cm}^{3}$, $\gamma^{\prime}=0.076 \mathrm{dyn} / \mathrm{cm} \cdot \mathrm{K}, \quad \mu=100 \mathrm{dyn} \cdot \mathrm{s} / \mathrm{cm}^{2}, \quad$ and $\alpha_{t}$ $=10^{-2} \mathrm{~cm}^{2} / \mathrm{s}$. It is noted that for $T_{g}=30 \mathrm{~K} / \mathrm{cm}$, a bubble of radius $0.2 \mathrm{~cm}$ would correspond to $\mathrm{Ma} \leqslant 0.1$ (see Ref. 12). More importantly, in the present analysis we pay attention only to the case in which the Reynolds and Marangoni numbers are ignored; otherwise, the resultant nonlinearity in equations makes the solutions too difficult to analyze.

In microgravity environment, the bubble migration is due to the thermal and hydrodynamic interactions. Hence, the equations of momentum and energy must be solved simultaneously. For the quasisteady case considered here, the governing equations are

$$
\begin{aligned}
& \nabla \cdot \mathbf{u}=0, \\
& \nabla p-\mu \nabla^{2} \mathbf{u}=0, \\
& \nabla^{2} T=0,
\end{aligned}
$$

where $p$ denotes the pressure and $\mathbf{u}$ the velocity in vector form. Here, the temperature field, $T=T_{g} z+T^{\prime}$, is the summation contributed by a constant temperature gradient far away from the bubbles and a disturbance temperature.

The boundary conditions to be satisfied on the surface of bubble $i(i=1,2)$ are

$$
\begin{aligned}
& \mathbf{u} \cdot \mathbf{n}_{i}=\mathbf{u}_{i} \cdot \mathbf{n}_{i}, \\
& \mathbf{n}_{i} \times\left(\boldsymbol{\sigma} \cdot \mathbf{n}_{i}+\nabla_{s} \gamma\right)=0, \\
& \mathbf{n}_{i} \cdot \nabla T=0,
\end{aligned}
$$

and the infinite conditions are

$$
\mathbf{u} \rightarrow 0 \quad \text { and } \nabla T \rightarrow T_{g} \mathbf{e}_{z} \quad \text { as } \quad \mathbf{r} \rightarrow \infty,
$$

where $\mathbf{u}_{i}$ is the migration velocity of bubble $i, \mathbf{n}_{i}$ an outward normal to the bubble, and $\boldsymbol{\sigma}$ represents the stress in the liquid, $\nabla_{s}$ the gradient along the surface of the bubble. Here, $\mathbf{e}_{z}$ is the unit vector pointing to the hotter portion of the liquid. In addition, for thermocapillary migration, if there are no 


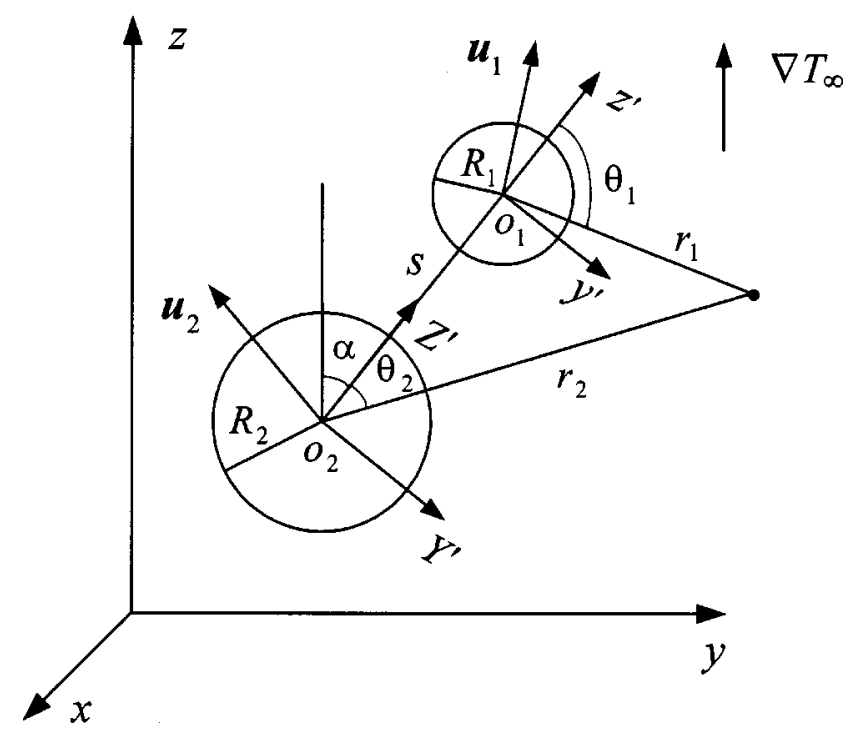

FIG. 1. Sketch of the two bubbles and the corresponding coordinate systems.

gravity or other nonhydrodynamic forces, the net hydrodynamic force on each bubble must be zero. This accordingly gives the following condition:

$$
\int_{\Sigma_{t}} \boldsymbol{\sigma} \cdot \mathbf{n}_{i} d s=0
$$

where $\Sigma_{i}$ denotes the surface of bubble $i$.

\section{ANALYTICAL SOLUTION}

To simplify analysis, two bubbles are considered to migrate along the $y-z$ symmetry plane, as shown in Fig. 1. In the reference frame $(x, y, z)$, the smaller of the two bubbles with center $o_{1}$ located at $\left(0, y_{1}, z_{1}\right)$ moves with a migration velocity $\mathbf{u}_{1}$ in the $y-z$ plane, whose components are $u_{11}$ and $u_{12}$, while the larger one with center $o_{2}$ situated at $\left(0, y_{2}, z_{2}\right)$ moves with a translational velocity $\mathbf{u}_{2}$ in the same plane, whose components are $u_{21}$ and $u_{22}$. The separation distance between the bubble centers $o_{1} o_{2}$ is denoted by $s$. In addition, a uniform temperature gradient, $\left|\nabla T_{\infty}\right|=T_{g}$, is imposed parallel to the $z$ axis.

For a two-bubble system, two other coordinates are introduced for convenience; one is a Cartesian coordinates $\left(x, y^{\prime}, z^{\prime}\right)$ fixed at the center of bubble 1 , the other, $\left(x, Y^{\prime}, Z^{\prime}\right)$, at the center of bubble 2. The instantaneous direction of the $z^{\prime}$ axis is chosen along the line joining the centers making an angle $\alpha$ with respect to the $z$ axis. $\mathbf{e}_{i}^{\prime}$ ( $i$ $=1,2,3)$ is the unit vector along the positive direction of the corresponding axis in the $\left(x, y^{\prime}, z^{\prime}\right)$ coordinates in alphabetical order. The translational transformation between $\left(x, y^{\prime}, z^{\prime}\right)$ and $\left(x, Y^{\prime}, Z^{\prime}\right)$ is

$$
\begin{aligned}
y^{\prime} & =Y^{\prime}, \\
z^{\prime} & =Z^{\prime}-s .
\end{aligned}
$$

These two Cartesian coordinate systems can be turned into spherical ones by

$$
\left\{\begin{array} { l } 
{ x = r _ { 1 } \operatorname { s i n } \theta _ { 1 } \operatorname { s i n } \varphi , } \\
{ y ^ { \prime } = r _ { 1 } \operatorname { s i n } \theta _ { 1 } \operatorname { c o s } \varphi , } \\
{ z ^ { \prime } = r _ { 1 } \operatorname { c o s } \theta _ { 1 } , }
\end{array} \text { and } \left\{\begin{array}{l}
x=r_{2} \sin \theta_{2} \sin \varphi, \\
Y^{\prime}=r_{2} \sin \theta_{2} \cos \varphi, \\
Z^{\prime}=r_{2} \cos \theta_{2} .
\end{array}\right.\right.
$$

From the general solution given by Lamb, ${ }^{16}$ the velocity field outside an isolated bubble of radius $R$ with migration velocity $u_{0}$ along the direction at an angle $\beta$ with respect to the $z^{\prime}$ axis is written as

$$
\begin{aligned}
\mathbf{u}= & -\frac{R^{3}}{2}\left[u_{01} \nabla\left(\frac{y^{\prime}}{r_{1}^{3}}\right)+u_{02} \nabla\left(\frac{z^{\prime}}{r_{1}^{3}}\right)\right]+\frac{1}{2 \mu}\left(R^{2}-\frac{r_{1}^{2}}{3}\right) \\
& \times\left[B_{01} \nabla\left(\frac{y^{\prime}}{r_{1}^{3}}\right)+B_{02} \nabla\left(\frac{z^{\prime}}{r_{1}^{3}}\right)\right]+\frac{2\left[B_{01} \nabla y^{\prime}+B_{02} \nabla z^{\prime}\right]}{3 \mu r_{1}},
\end{aligned}
$$

where $u_{01}=u_{0} \sin \beta$ and $u_{02}=u_{0} \cos \beta$. Here, $B_{01}$ and $B_{02}$ are two constants to be determined by the boundary condition.

Similarly, neglecting the normal flux of energy, the temperature field outside the same bubble in the $\left(x, y^{\prime}, z^{\prime}\right)$ coordinates is

$$
T=T_{g}\left(1+\frac{R^{3}}{2 r_{1}^{3}}\right)\left(-y^{\prime} \sin \alpha+z^{\prime} \cos \alpha\right) .
$$

According to the superposition principle for linear equations, we construct the velocity field $\mathbf{u}$ in surrounding fluid from (5) using the fundamental solutions of velocity field for a two-bubble system (see the Appendix), that is,

$$
\begin{aligned}
\mathbf{u}= & \sum_{m=0}^{1}\left(-\frac{u_{1,2-m}^{\prime} R_{1}^{3}}{2}+\frac{B_{1,2-m} R_{1}^{2}}{2 \mu}\right) \mathbf{h}_{\mathbf{m}}^{\prime}-\frac{B_{1,2-m} \mathbf{h}_{\mathbf{m}}^{\prime \prime}}{6 \mu} \\
& +\frac{2 B_{1,2-m} \mathbf{h}_{\mathbf{m}}^{\prime \prime \prime}}{3 \mu}+\left(-\frac{u_{2,2-m}^{\prime} R_{2}^{3}}{2}+\frac{B_{2,2-m} R_{2}^{2}}{2 \mu}\right) \mathbf{H}_{\mathbf{m}}^{\prime} \\
& -\frac{B_{2,2-m} \mathbf{H}_{\mathbf{m}}^{\prime \prime}}{6 \mu}+\frac{2 B_{2,2-m} \mathbf{H}_{\mathbf{m}}^{\prime \prime \prime}}{3 \mu} .
\end{aligned}
$$

In this expression, $u_{i j}^{\prime}(i, j=1,2)$ denote the migration velocity components of the two bubbles in the $\left(x, y^{\prime}, z^{\prime}\right)$ coordinates. And, the temperature distribution $T$ constructed in the same way from (6) is written as

$$
T=T_{g} z+\frac{T_{g}}{2} \sum_{m=0}^{1} \cos (\alpha+m \pi / 2)\left(R_{1}^{3} t_{m}^{\prime}+R_{2}^{3} \tau_{m}^{\prime}\right) .
$$

Substituting $\mathbf{u}$ and $T$ aforementioned into (2b) and (2e) leads, with manipulation, to eight algebraic equations of $u_{i j}^{\prime}$ and $B_{i j}(i, j=1,2)$. In microgravity environment, $g \rightarrow 0$ inevitably results in $B_{i j} \rightarrow 0$. Therefore, the total number of the equations in the present case is reduced to four, which in the $\left(x, y^{\prime}, z^{\prime}\right)$ coordinate system finally become

$$
\begin{aligned}
& \frac{u_{i 2}^{\prime}}{R_{i}}\left\{1+\frac{R_{i}^{3}}{2} \sum_{j=0}^{\infty} \lambda_{i 01}^{(2 j)}\right\}+\frac{u_{3-i, 2}^{\prime} R_{3-i}^{3}}{2 R_{i}} \sum_{j=0}^{\infty} \lambda_{3-i, 01}^{(2 j+1)} \\
& \quad-\frac{\gamma^{\prime} T_{g} \cos \alpha}{2 \mu}\left\{1+\frac{R_{i}^{3}}{2} \sum_{j=0}^{\infty} \phi_{i 01}^{(2 j)}+\frac{R_{3-i}^{3}}{2} \sum_{j=0}^{\infty} \phi_{3-i, 01}^{(2 j+1)}\right\}=0
\end{aligned}
$$

for $i=1$ and 2 , 


$$
\begin{aligned}
& \frac{u_{i 1}^{\prime}}{R_{i}}\left\{1+\frac{R_{i}^{3}}{2} \sum_{j=0}^{\infty} \lambda_{i 11}^{(2 j)}\right\}+\frac{u_{3-i, 1}^{\prime} R_{3-i}^{3}}{2 R_{i}} \sum_{j=0}^{\infty} \lambda_{3-i, 11}^{(2 j+1)} \\
& +\frac{\gamma^{\prime} T_{g} \sin \alpha}{2 \mu}\left\{1+\frac{R_{i}^{3}}{2} \sum_{j=0}^{\infty} \phi_{i 11}^{(2 j)}+\frac{R_{3-i}^{3}}{2} \sum_{j=0}^{\infty} \phi_{3-i, 11}^{(2 j+1)}\right\}=0 \\
& \text { for } i=1 \text { and 2. }
\end{aligned}
$$

Here, $u_{i j}^{\prime}(i, j=1,2)$ may be transformed into $u_{i j}(i, j=1,2)$ using

$$
\left\{\begin{array}{l}
u_{i 1}^{\prime}=u_{i 1} \cos \alpha-u_{i 2} \sin \alpha, \\
u_{i 2}^{\prime}=u_{i 1} \sin \alpha+u_{i 2} \cos \alpha,
\end{array} \quad i=1,2 .\right.
$$

One can get well-known results, $u_{i 2}=\gamma^{\prime} T_{g} R_{i} /(2 \mu)$ and $u_{i 1}=0(i=1,2)$, by letting $s \rightarrow \infty$ in Eqs. (7a) and (7b) since all interaction coefficients related to $s$ approach zero.

On the surfaces of two bubbles, the heat energy is transformed into the kinetic one of the fluid around them. The kinetic energy $E_{k}$ of the whole flow field is given below

$$
\begin{aligned}
E_{k}= & \frac{\rho}{2} \int_{v_{f}} \mathbf{u}^{\prime} \cdot \mathbf{u}^{\prime} d v \\
= & \pi \rho \sum_{i=1}^{2} \sum_{k=1}^{2} R_{k}^{3}\left(\frac{u_{k i}^{\prime 2}}{3}\left[1+\frac{3 R_{k}^{3}}{2} \sum_{j=0}^{\infty} \lambda_{k, 2-i, 1}^{(2 j)}\right]\right. \\
& \left.+\frac{u_{k i}^{\prime} u_{3-k, i}^{\prime} R_{3-k}^{3}}{2} \sum_{j=0}^{\infty} \lambda_{k, 2-i, 1}^{(2 j+1)}\right),
\end{aligned}
$$

where $v_{f}$ denotes the whole region occupied by the fluid.

\section{RESULTS}

This investigation presents analytical expressions for the thermocapillary migration velocities of two bubbles. This is a main contribution of the present work. The algebraic equations of the bubble migration velocity, (7a) and (7b), are applied to determine the interaction between two bubbles and predict their migrations in a microgravity environment. To this end, the numerical calculation is the vital resort of determining both the migration velocities of two bubbles and their trajectories. As each of the $m$ th terms in recurrence formulas (A7), (A13), (A19), and (A21) in the Appendix behaves like $1 / s^{3 m}$, the truncated series in the above formulas at $m=50$ would have errors smaller than the error tolerance of six significant figures even for a near contact. The series in Eqs. (7a) and (7b) truncated at $j=50$ makes our numerical results accurate up to the 300th inverse power of $s$ in the present case. To assure convergence, our calculations are carried out with an automatic increase in $j$ until the summation of extra terms produces no significant change within the desired level of accuracy. Furthermore, in order to determine the trajectories of the two-bubble migration, integration of Eqs. (7a) and (7b) with respect to time is performed using the fourth-order Runge-Kutta-Fehlberg method. ${ }^{17}$ The computation is started at the initial positions $y_{1}(0)=y_{10}, z_{1}(0)$ $=z_{10}, y_{2}(0)=y_{20}$, and $z_{2}(0)=z_{20}$. The accuracy of the integration is checked by halving the $k$ th time step $\delta t_{k}(k$ $=1,2 \ldots)$ and then comparing the value of the fourth-order Runge-Kutta integration with the fifth one at that instant so
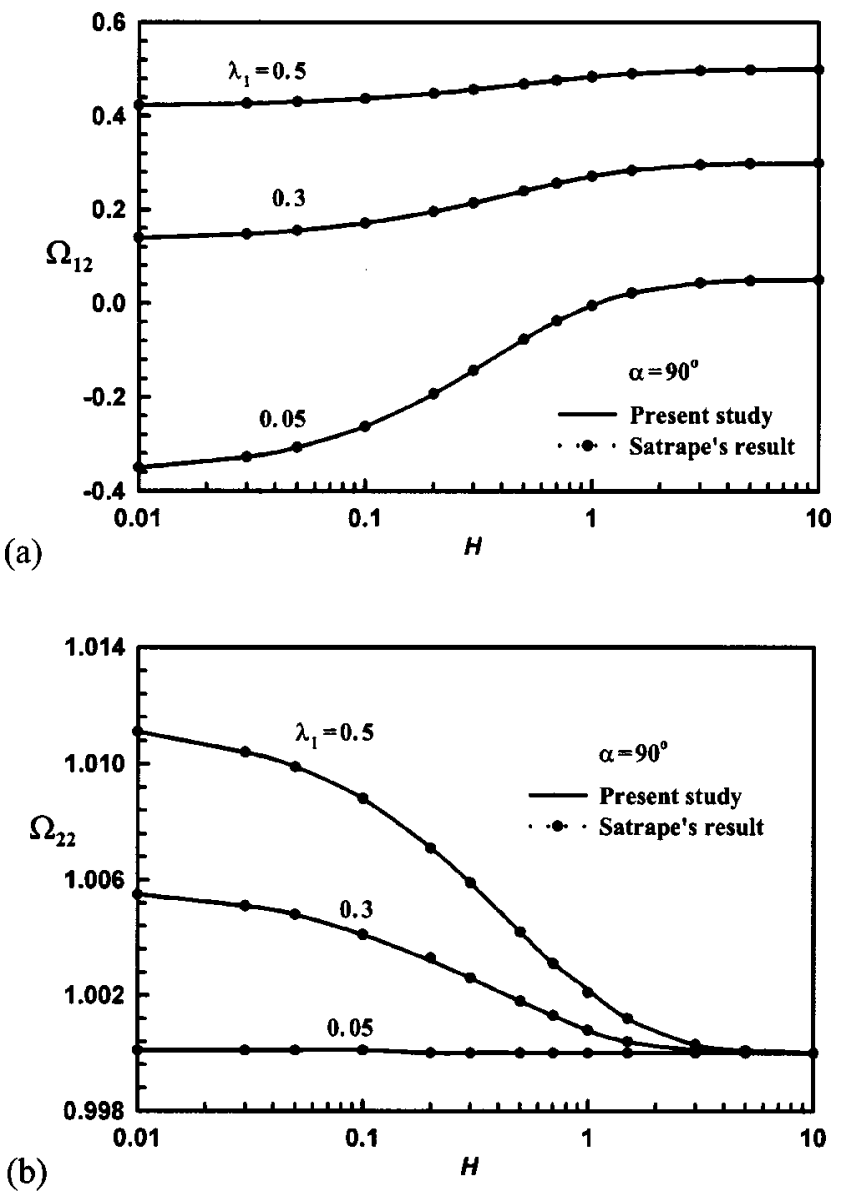

FIG. 2. Interaction parameters for two bubbles aligned perpendicular to the applied thermal gradient. Curves are plotted for both the (a) smaller bubbler and (b) larger bubbles.

that the $k$ th results converge within a preassigned limit (1.0 $\left.\times 10^{-6}\right)$. This process is repeated until either two bubbles collide with each other or one bubble overtakes the other. Thus, given the initial locations, we can determine whether or not the two bubbles will eventually collide in their subsequent motions.

\section{A. Results of the interactions}

To better represent thermal migration velocities of the two bubbles, we have to define the interaction parameter as in Ref. 4 with a little alteration

$$
\begin{aligned}
& H=\frac{s-R_{1}-R_{2}}{R_{2}}, \\
& \lambda_{i}=\frac{R_{i}}{R_{2}}, \\
& \Omega_{i j}\left(\alpha, H, \lambda_{i}\right)=\frac{u_{i j}}{u_{2 \mathrm{YGB}}}, \quad i, j=1,2,
\end{aligned}
$$

where $u_{2 \mathrm{YGB}}=\gamma^{\prime} T_{g} R_{2} /(2 \mu)$. Here, $\Omega_{i j}$ is a measure of the influence of the second bubble on the migration velocity of the first one.

In Fig. 2, some of our results for bubbles aligned perpendicular to the imposed thermal gradient, $\alpha=90^{\circ}$, are com- 
(a)
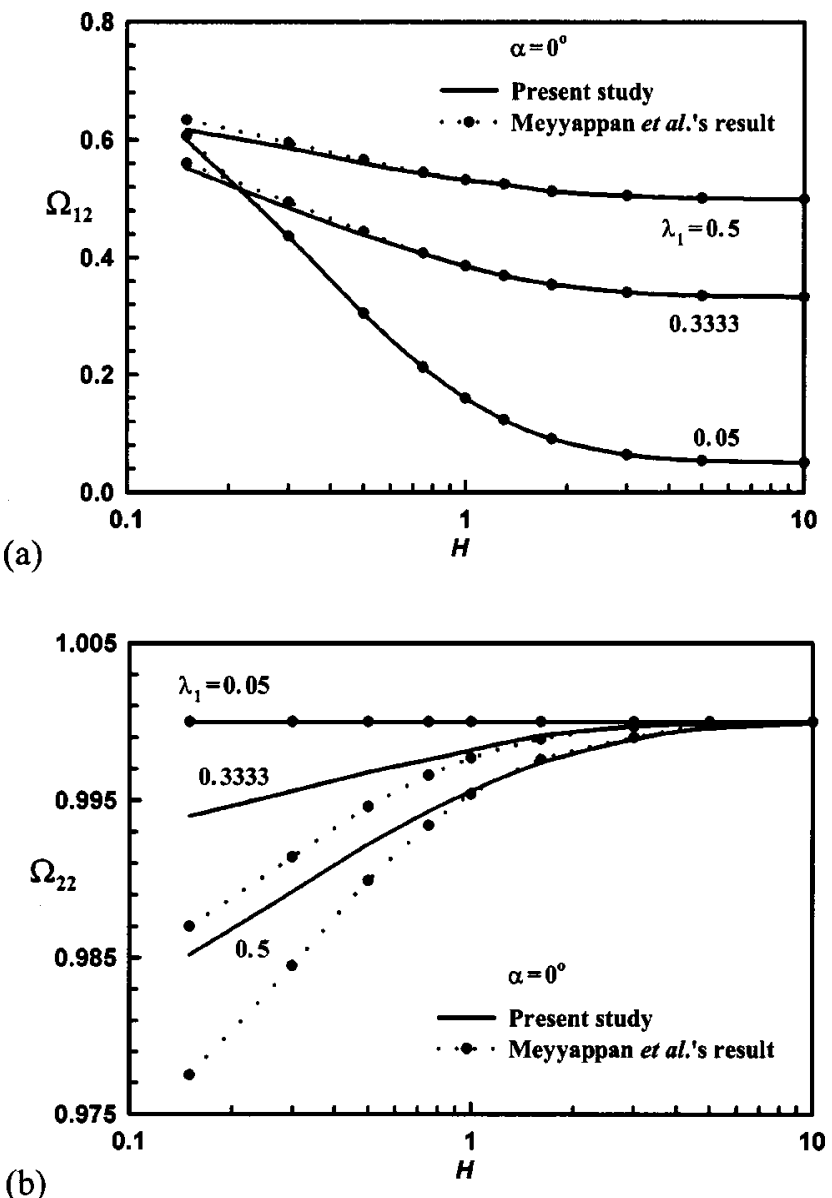

FIG. 3. Interaction parameters for two bubbles aligned parallel to the applied thermal gradient. Curves are plotted for both the (a) smaller bubbler and (b) larger bubbles.

pared to ones given by Satrape. ${ }^{12}$ For completeness, some results for two bubbles aligned parallel to the applied thermal gradient, $\alpha=0^{\circ}$, are also shown graphically in Fig. 3 in comparison with those given by Meyyappan et al. ${ }^{4}$ As shown in Fig. 2, agreement between two sets of results is quite good, and all corresponding curves almost coincide with each other. Numerical computation shows that our results are in quantitative agreement with those of Satrape and Wang et al. ${ }^{13}$ up to four significant figures for both the whole range of separation distance and various size ratios. Note from Fig. 3 that for the corresponding size ratios, $\lambda_{1}=0.05,0.3333$, and 0.5 , our results for the case of the smaller bubble agree well with ones presented in Ref. 4, but for the case of the larger bubble, there is the largest difference less than $1 \%$ as the two bubbles are coming into contact. The reason may be that these two solutions are expressed in terms of two kinds of functions, respectively, hyperbolic functions and power ones, and thus there are different accumulative errors in each individual calculation, especially for rather small separation distances. But, this slight difference is unrelated to the description of the physical process.

After comparison, let us next consider the planar thermocapillary migration of two identical bubbles. In the light of Eqs. (7a), (7b), and (8), we find that the two equal-sized bubbles move along the temperature gradient $\nabla T_{\infty}$ at the velocity that they would have as if they were in isolation, independent of the dimensionless gap $H$ and orientation angle $\alpha$. This is an extension of the conclusion presented by Meyyappan et al. ${ }^{4}$ and Feuillebois ${ }^{5}$ to the case of arbitrary configuration. The following is a proof of it: as $R_{1}=R_{2}$ $=R$, Eqs. (7a) and (7b) are rewritten as

$$
\begin{array}{r}
u_{i 2}^{\prime}\left\{1+\frac{R^{3}}{2} \sum_{j=0}^{\infty} \lambda_{i 01}^{(2 j)}\right\}+\frac{u_{3-i, 2}^{\prime} R^{3}}{2} \sum_{j=0}^{\infty} \lambda_{3-i, 01}^{(2 j+1)} \\
-\frac{\gamma^{\prime} T_{g} R \cos \alpha}{2 \mu}\left\{1+\frac{R^{3}}{2} \sum_{j=0}^{\infty} \phi_{i 01}^{(2 j)}+\frac{R^{3}}{2} \sum_{j=0}^{\infty} \phi_{3-i, 01}^{(2 j+1)}\right\}=0 \\
\text { for } i=1 \quad \text { and 2, }
\end{array}
$$

and

$$
\begin{array}{r}
u_{i 1}^{\prime}\left\{1+\frac{R^{3}}{2} \sum_{j=0}^{\infty} \lambda_{i 11}^{(2 j)}\right\}+\frac{u_{3-i, 1}^{\prime} R^{3}}{2} \sum_{j=0}^{\infty} \lambda_{3-i, 11}^{(2 j+1)} \\
+\frac{\gamma^{\prime} T_{g} R \sin \alpha}{2 \mu}\left\{1+\frac{R^{3}}{2} \sum_{j=0}^{\infty} \phi_{i 11}^{(2 j)}+\frac{R^{3}}{2} \sum_{j=0}^{\infty} \phi_{3-i, 11}^{(2 j+1)}\right\}=0 \\
\text { for } i=1 \text { and } 2 .
\end{array}
$$

Based on iterative formulas (A7), (A13), (A19), and (A21) in the Appendix, it is easy to find that

$$
\sum_{j=0}^{\infty} \lambda_{i m k}^{(2 j)}=\sum_{j=0}^{\infty} \phi_{i m k}^{(2 j)}
$$

and

$$
\sum_{j=0}^{\infty} \lambda_{i m k}^{(2 j+1)}=\sum_{j=0}^{\infty} \phi_{i m k}^{(2 j+1)},
$$

$$
i=1,2 ; \quad m=0,1 ; \quad k=1,2,3 \ldots .
$$

Thus, we obtain $\quad u_{i 1}^{\prime}=-\gamma^{\prime} T_{g} R \sin \alpha /(2 \mu) \quad$ and $\quad u_{i 2}^{\prime}$ $=\gamma^{\prime} T_{g} R \cos \alpha /(2 \mu)(i=1,2)$ by solving (13a) and (13b). Substituting these expressions into (8), one can derive immediately $u_{i 2}=\gamma^{\prime} T_{g} R /(2 \mu)$ and $u_{i 1}=0$ in any array. This implies that the thermal and fluid mechanical interaction effects are counterbalanced thoroughly in the two-identical-bubble case.

From Eqs. (7a) and (7b), we can combine the perpendicular cases with the parallel ones to obtain corresponding cases for bubbles at arbitrary orientations. Figures 4 and 5 illustrate sample results for two bubbles with designated orientations of $\alpha=45^{\circ}$ and $\alpha=135^{\circ}$, respectively. It is observed from these figures that the interaction between two bubbles has rather significant influence on the planar migration of the smaller bubble but a comparatively weak effect on the larger one. The larger the size ratio of the larger bubble to the smaller one is, the greater the variation in the velocity of the smaller one. Furthermore, it is seen from Figs. 4 and 5 that the migration velocities of the two bubbles have opposite signs in the $y$ direction. This means that the two bubbles try to push each other away, with the larger bubble pursuing the smaller one, and pull each other back after the larger bubble overtakes the smaller one. It is also seen from the figures that 
(a)
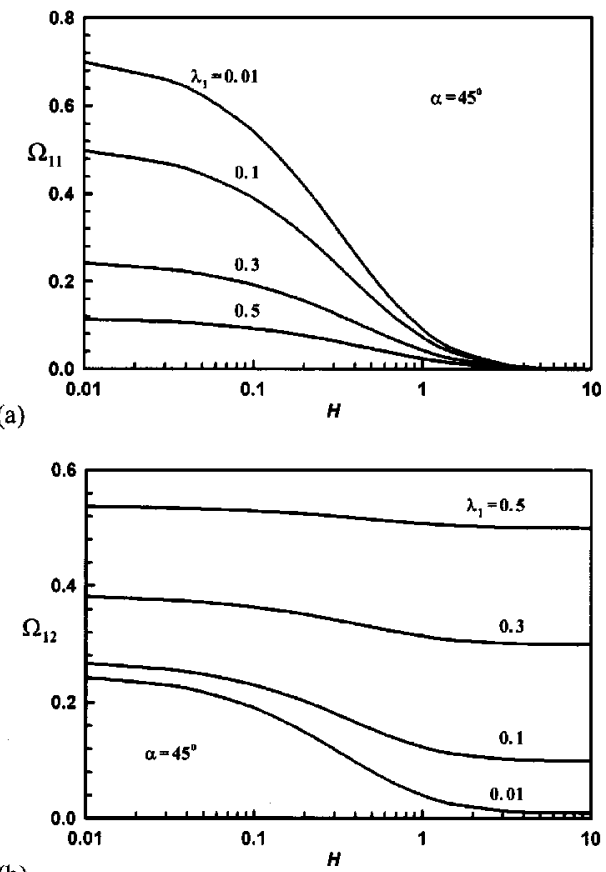

(b)

FIG. 4. Interaction parameters for two bubbles with various bubble size ratios at $\alpha=45^{\circ}$.
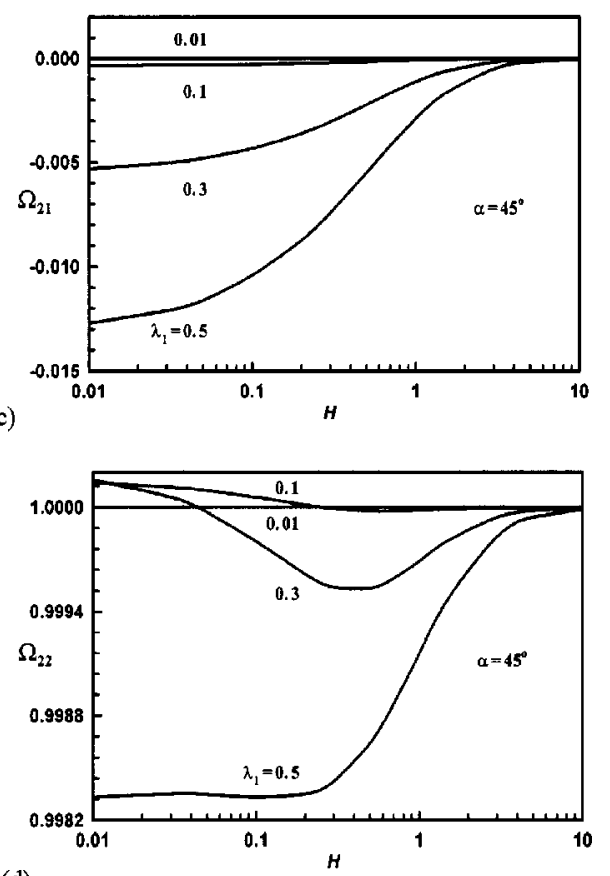

(d) the migration velocities of each bubble in the two designated configurations have the same magnitude but opposite components in the $y$ direction. These can be intuitively interpreted as a consequence of the reversibility of the Stokes flow. On the other hand, the velocity component of the larger bubble in the direction parallel to the imposed thermal gradient does not always diminish monotonically with the decrease in the separation distance, but increases in some intervals for such cases as $\lambda_{1}=0.1$ and $\lambda_{1}=0.3$. Typical plots are depicted in Figs. 4(d) and 5(d). A conclusion can be drawn from Figs. 4 and 5 that in the presence of other bubbles, a larger bubble would even migrate beyond its typical speed in the isolated situation.

When a bubble approaches another very small one during migration, the velocity of the smaller bubble will change greatly. The question arises as to what would happen when the two bubbles touch each other without coalescence. Here is the answer that can be inferred from Eqs. (7a) and (7b): as

(c)

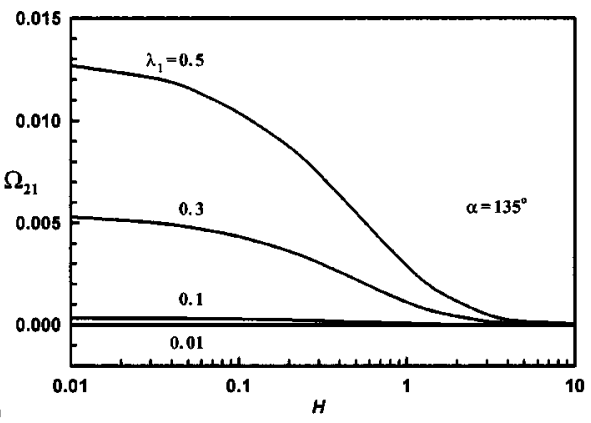

(a)
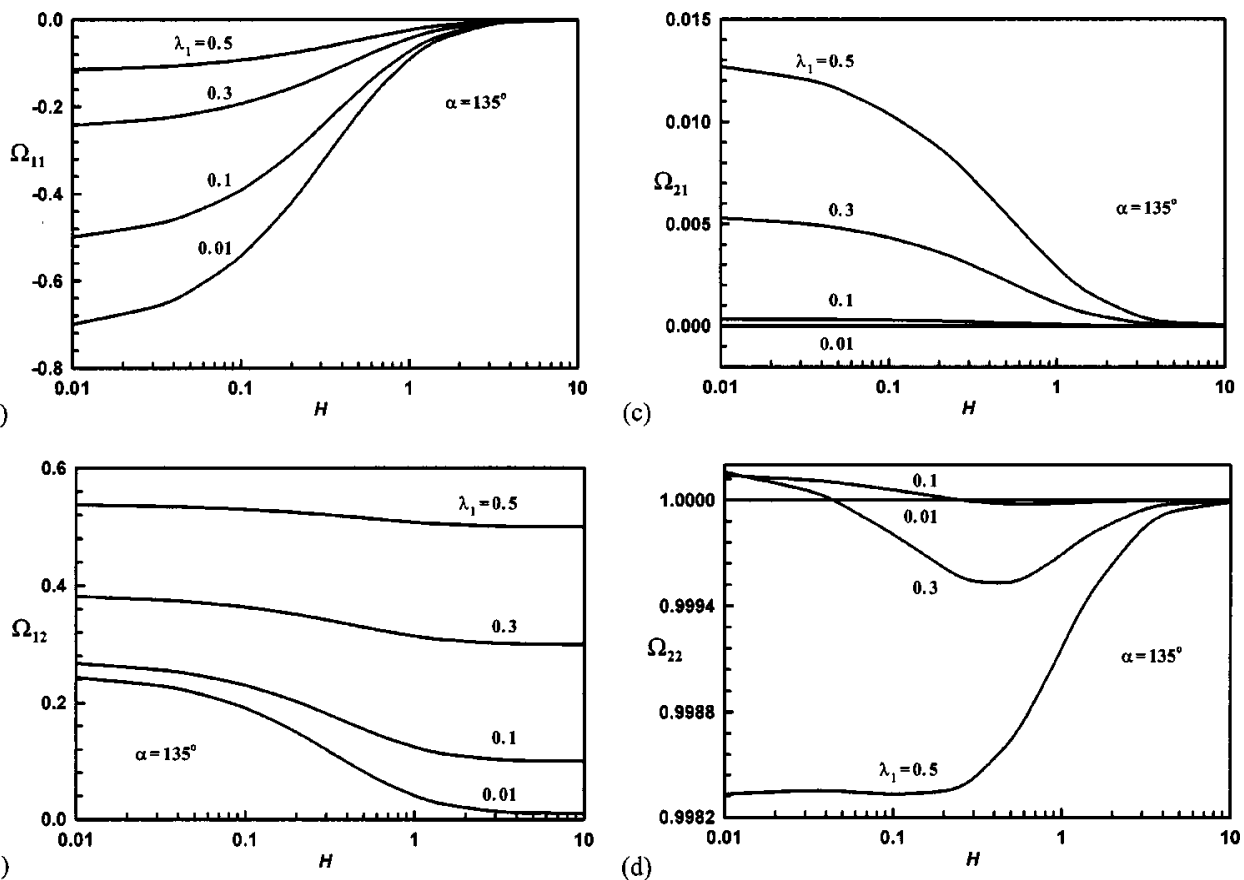

FIG. 5. Interaction parameters for two bubbles with various bubble size ratios at $\alpha=135^{\circ}$. 
$R_{1} \rightarrow 0$ and $s \rightarrow R_{1}+R_{2} \rightarrow R_{2}$, (7a) and (7b) can be simplified as

$$
\begin{aligned}
& u_{12}^{\prime}-u_{22}^{\prime}+O\left(R_{1}\right)=0, \\
& u_{22}^{\prime}-u_{2 \mathrm{YGB}} \cos \alpha+o\left(R_{1}\right)=0, \\
& u_{11}^{\prime}+\frac{u_{21}^{\prime}}{2}+O\left(R_{1}\right)=0,
\end{aligned}
$$

and

$$
u_{21}^{\prime}+u_{2 \mathrm{YGB}} \sin \alpha+o\left(R_{1}\right)=0 .
$$

That is, $\quad u_{12}^{\prime} \approx u_{22}^{\prime} \approx u_{2 \mathrm{YGB}} \cos \alpha \quad$ and $\quad u_{21}^{\prime} \approx-2 u_{11}^{\prime}$ $\approx-u_{2 \mathrm{YGB}} \sin \alpha$. Therefore, $u_{12}^{\prime}$ reaches a maximum of $u_{2 \mathrm{YGB}}$ as $\alpha=0^{\circ}$ and $u_{11}^{\prime}$ equals $u_{2 \mathrm{YGB}} / 2$ as $\alpha=90^{\circ}$, and there is a relationship between $u_{11}^{\prime}$ and $u_{12}^{\prime}, u_{12}^{\prime 2}+4 u_{11}^{\prime 2}=u_{2 \mathrm{YGB}}^{2}$. In the present situation, the $\left(x, Y^{\prime}, Z^{\prime}\right)$ coordinates with the origin at $o_{2}$ are approximately an inertial frame of reference. Relative to this moving reference frame, the velocity components of the tiny bubble are $U_{12}^{\prime}=u_{12}^{\prime}-u_{22}^{\prime}=0$ and $U_{11}^{\prime}$ $=u_{11}^{\prime}-u_{21}^{\prime}=3 u_{2 \mathrm{YGB}} \sin \alpha / 2$, and hence the tiny bubble will travel on the larger bubble surface along the $y^{\prime}$ direction. Having recourse to the stability analysis, one can obtain not only the equilibrium points but also some information on the behavior of the tiny bubble on the surface of the larger one. There are two stationary solutions of the two differential equations mentioned above, one at $\alpha=0^{\circ}$ which is unstable and the other at $\alpha=180^{\circ}$ which is stable. An intuitive interpretation is that when a migrating tiny bubble gets in touch with another larger bubble without coalescence, it will move on the surface of the larger one until reaching the position at $\alpha=180^{\circ}$.

On the other hand, in the same situation, heuristic approximations

$$
\frac{u_{12}^{\prime}}{u_{1 \mathrm{YGB}}}=\left\{1+\left(\frac{R_{2}}{s}\right)^{3}\left(\frac{R_{2}}{R_{1}}-1\right)\right\} \cos \alpha,
$$

and

$$
\frac{u_{11}^{\prime}}{u_{1 \mathrm{YGB}}}=-\left\{1-0.5\left(\frac{R_{2}}{s}\right)^{3}\left(\frac{R_{2}}{R_{1}}-1\right)\right\} \sin \alpha,
$$

can be derived directly from (7a) and (7b). They give a corresponding expression to that of Meyyappan et al. ${ }^{4}$ as $\alpha=0^{\circ}$.

And even more important, it is observed from the above figures that as the dimensionless gap $H$ is less than 2.0, the migration velocity of the smaller bubble varies greatly due to interaction. The physical intuition suggests that there is an "interaction region" around a bubble, which is defined as one diameter apart from the bubble. If a second smaller bubble rushes into the interaction region of the first one, the first bubble would significantly affect the subsequent migration of the smaller one.

\section{B. Bubble trajectories}

Trajectories of two nearby bubbles can be obtained by numerically integrating in time Eqs. (7a) and (7b). One is

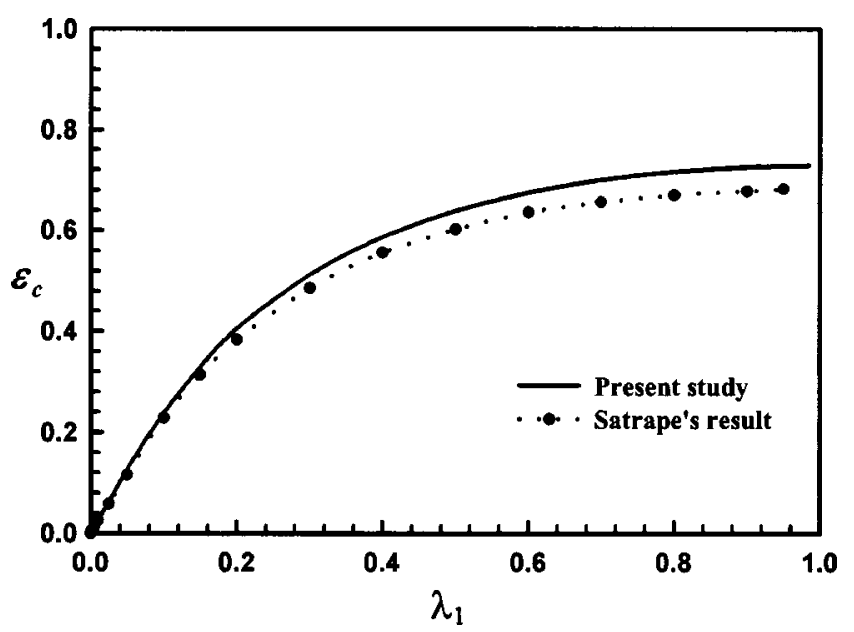

FIG. 6. Collision efficiency $\varepsilon_{c}$ against bubble size ratio $\lambda_{1}$.

thereby able to predict both a potential collision of the two bubbles during the thermocapillary migration and describe their dynamic behaviors.

For the study of bubble interaction, it is important to foretell whether or not a migrating bubble can impact with another one, so a useful application of trajectory calculations for two bubbles is the determination of collision efficiency $\varepsilon_{c}$. Following Satrape's definition, we define collision efficiency as the ratio of the minimal cross-sectional area of the body of revolution formed by the limiting trajectory to $\pi\left(R_{1}+R_{2}\right)^{2}$. On can deduce easily from Eqs. (7a), (7b), and (8) that for any two correlative orientation cases, $\alpha$ and $180^{\circ}-\alpha, \quad u_{i 2}\left(180^{\circ}-\alpha\right)=u_{i 2}(\alpha)$ and $u_{i 1}\left(180^{\circ}-\alpha\right)$ $=-u_{i 1}(\alpha)$, i.e., trajectories of the two bubbles are all symmetric with respect to $\alpha=90^{\circ}$. By making use of the consequence, we obtain the limiting trajectories in the same manner as Ref. 12: two bubbles are released initially perpendicular to the thermal gradient and just in contact, and then their motion is integrated until each bubble once again gains a velocity when isolated. The collision efficiency against the size ratio $\lambda_{1}$ are plotted in Fig. 6 and compared with those given by Satrape. Note from Fig. 6 that the difference between the two sorts of results is not large. Our results are slightly larger than those presented by Satrape.

Figures 7, 8, and 9 give examples to better understanding of how a larger bubble pursues a smaller one. In these sample cases, $R_{1}=0.05 \mathrm{~cm}, R_{2}=0.5 \mathrm{~cm}, \gamma^{\prime}=0.076 \mathrm{dyn} /$ $\mathrm{cm} \cdot \mathrm{K}, T_{g}=30 \mathrm{~K} / \mathrm{cm}$, and $\mu=100 \mathrm{dyn} \cdot \mathrm{s} / \mathrm{cm}^{2}$, corresponding to a situation of two bubbles in a borax glass melt. All displacements here are nondimensionalized with respect to $R_{2}$. One will find interesting results from these figures: there are three typical dynamical behaviors in two-bubble planar migration, which correspond to three initially released positions of bubble 1 , that is $\left(y_{10} / R_{2}, z_{10} / R_{2}\right)=(0.58,4.1)$, $(1.505,4.1)$, and $(6.533,18)$, respectively, while bubble 2 is always set free at origin. It is observed that the trajectory of the smaller bubble appears to be a folium pattern in shape in Fig. 7(a), a cissoid pattern in Fig. 8(a), and a witch pattern in Fig. 9(a). This implies that the initial array of two bubbles greatly affects the subsequent migration of the smaller bubble. Obviously, the state parameter in these cases is the 
(a)
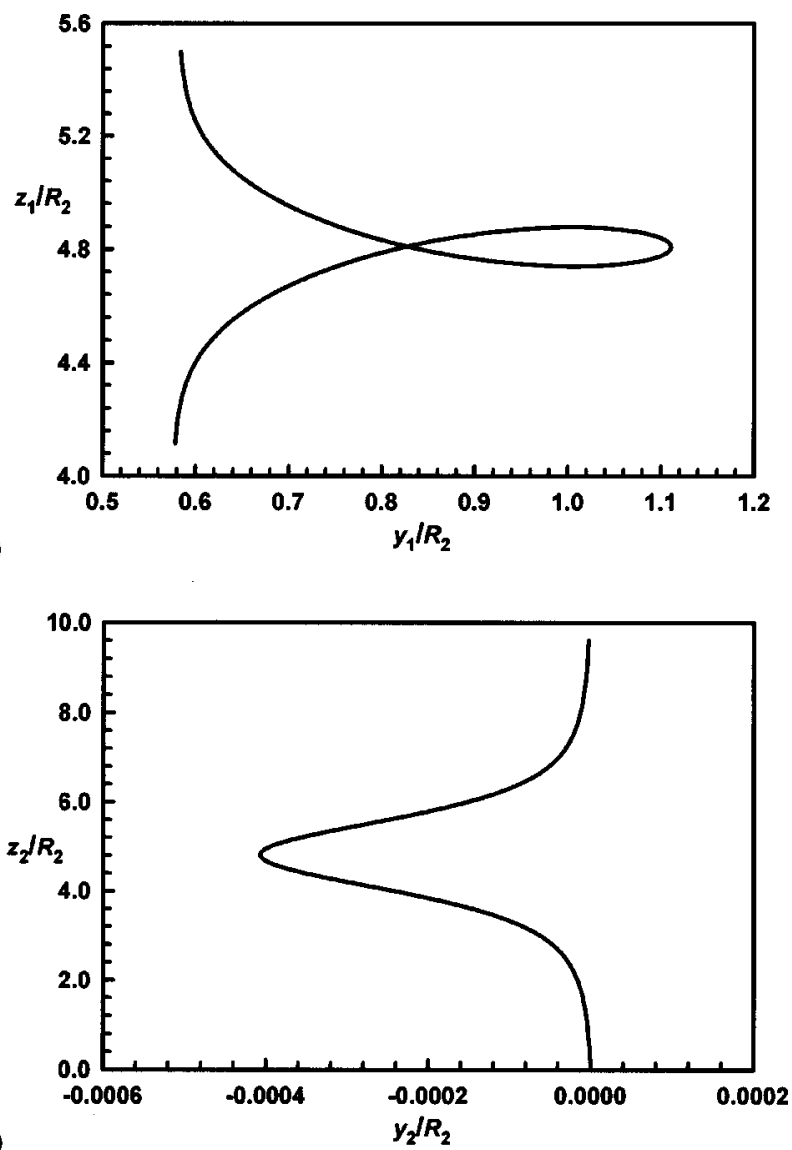

(b)

FIG. 7. Motions of two bubbles with $R_{1}=0.05 \mathrm{~cm}, R_{2}=0.5 \mathrm{~cm}, \gamma^{\prime}=0.076$ dyn $/ \mathrm{cm} \cdot \mathrm{K}, T_{g}=30 \mathrm{~K} / \mathrm{cm}$, and $\mu=100 \mathrm{dyn} \cdot \mathrm{s} / \mathrm{cm}^{2}$ for the initially released positions $y_{10} / R_{2}=0.58$ and $z_{10} / R_{2}=4.1$.

initial transverse position $y_{10}$ of the smaller bubble relative to the larger one. As seen from Figs. 7(a), 8(a), and 9(a), as the value of the parameter is less than a critical threshold, the trajectory of the smaller bubble demonstrates a foliumpattern motion. It shows a cissoidal behavior at the critical point, and then further becomes a witch pattern in shape with the initial transverse position being beyond the limit. These phenomena may be explained as follows: as the larger bubble pursues the smaller one in front of it, its flow field pushes the smaller bubble ahead. As the larger bubble leaves the smaller one behind, its wake draws the smaller bubble toward itself. But, the flow field of the larger bubble carries the smaller one backwards when the two bubbles migrate side by side. Thus, when a tiny bubble near a large one migrates in the neighborhood of $\alpha=90^{\circ}$, it is possible that the speed of fluid around the tiny bubble is greater than the relative speed of the tiny bubble to the fluid so that the flow compels the tiny bubble to move in the direction opposite to its migration [see Fig. 2(a)]. This suggests that in this situation, the larger bubble at first approaches the smaller one and drives it aside, and at some time when the two bubbles migrate in the proximity of $\alpha=90^{\circ}$, the smaller bubble gradually decreases its speed to zero and even moves backwards. Finally, the larger bubble overtakes the smaller one and then the reverse process occurs-we shall see the first moving pattern accordingly. If one adjusts the initial transverse posi-

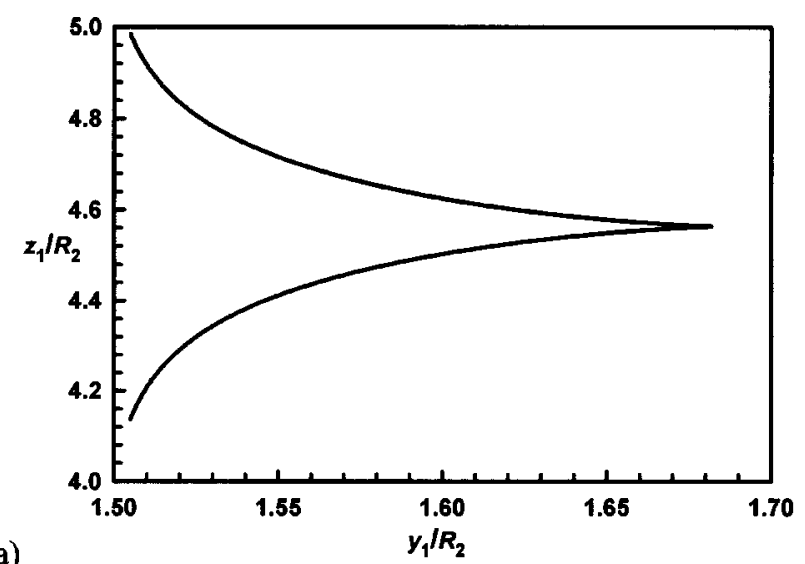

(a)

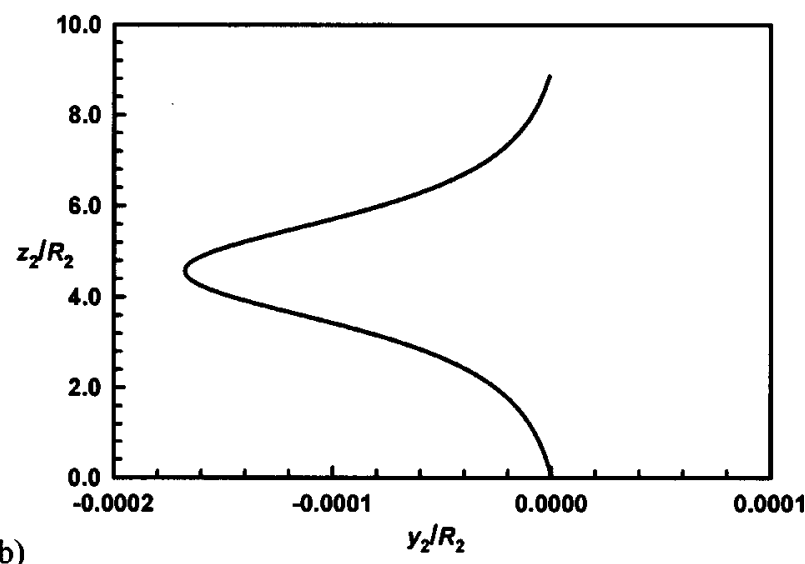

(b)

FIG. 8. Motions of two bubbles with $R_{1}=0.05 \mathrm{~cm}, R_{2}=0.5 \mathrm{~cm}, \gamma^{\prime}=0.076$ $\mathrm{dyn} / \mathrm{cm} \cdot \mathrm{K}, T_{g}=30 \mathrm{~K} / \mathrm{cm}$, and $\mu=100 \mathrm{dyn} \cdot \mathrm{s} / \mathrm{cm}^{2}$ for the initially released positions $y_{10} / R_{2}=1.505$ and $z_{10} / R_{2}=4.1$.

tion $y_{10}$ to such a configuration that the velocity of the smaller bubble is just zero as $\alpha=90^{\circ}$, he will acquire the second moving pattern. Further increasing $y_{10}$ would lead to the occurrence of the last pattern. In computation, we note that not all the smaller bubble with various size ratios may display a folium-pattern behavior. Generally, enlarging the size ratio $\lambda_{1}$ would lead "the strophoid" to shrink and move itself to the side of the large bubble. It is intuitively clear that there is a critical size ratio $\lambda_{1 c r}$ for the moving pattern. Using the trial-and-error method, we obtain the critical value of $\lambda_{1 c r}=0.22056$. A smaller bubble with such a size ratio, when set in contact with another larger one and aligned with it perpendicular to the imposed thermal gradient, is just at a standstill at that instant. As a result, a small bubble with a size ratio $\lambda_{1}>\lambda_{1 c r}$ would not demonstrate a folium-pattern motion at all. On the other hand, contrasting 7(a) with 7(b), 8(a) with 8(b), and 9(a) with 9(b), respectively, shows that protrusions of the three pairs of curves point towards opposite directions. A phenomenological explanation is that two bubbles in planar migration push each other as the larger bubble approaches the smaller one and then pull each other after the larger bubble overtakes the smaller one until they are once again far apart. Each of the two bubbles in the whole migration will be virtually in the same place as they were isolated, except for the region where the interaction cannot be ignored. Furthermore, the kinetic energy $E_{k}$ of the 


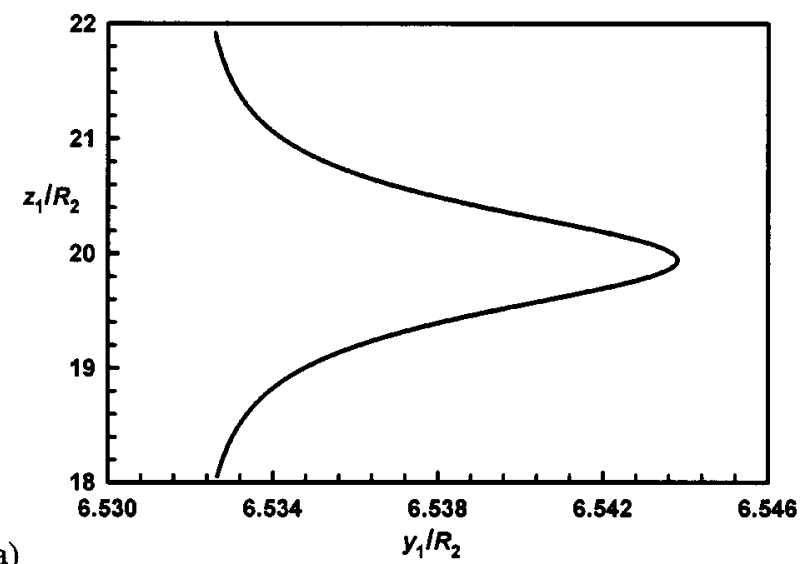

(a)

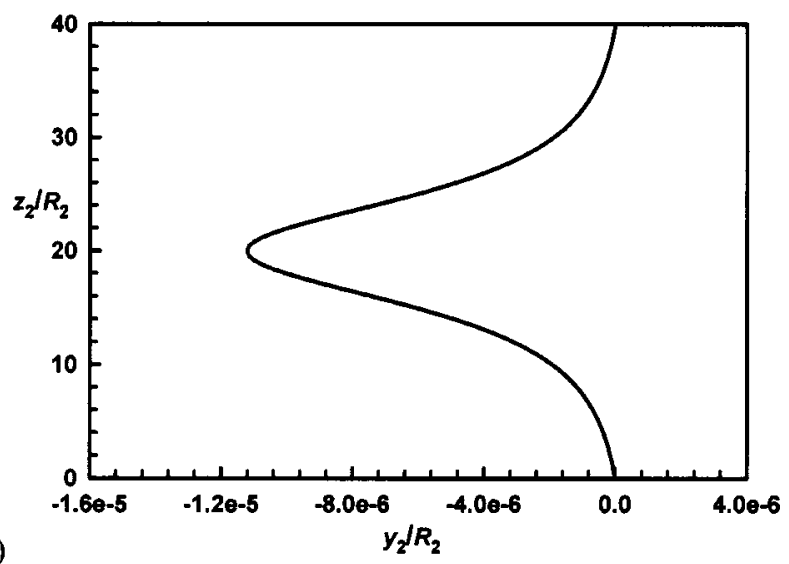

(b)

FIG. 9. Motions of two bubbles with $R_{1}=0.05 \mathrm{~cm}, R_{2}=0.5 \mathrm{~cm}, \gamma^{\prime}=0.076$ dyn $/ \mathrm{cm} \cdot \mathrm{K}, T_{g}=30 \mathrm{~K} / \mathrm{cm}$, and $\mu=100 \mathrm{dyn} \cdot \mathrm{s} / \mathrm{cm}^{2}$ for the initially released positions $y_{10} / R_{2}=6.533$ and $z_{10} / R_{2}=18$.

flow around the two bubbles would change because the interaction between them alters the transformation of the heat energy into the kinetic one on the two bubble surfaces. The kinetic energy of the fluid can be easily obtained from Eqs. (7a), (7b), and (9), and the results are plotted in Fig. 10 in dimensionless form. Here, $E_{k \mathrm{o}}$ denotes the total kinetic energy of the fluid in the isolated cases. It is seen from Fig. 10 (a) that in the axisymmetric situation, the kinetic energy of the fluid lessens with the decrease in the separation distance or increase in the size ratio $\lambda_{1}$ of the two bubbles, and it reaches its minimum of about $71 \%$ of $E_{k \mathrm{o}}$ as the two touching bubbles have the same size, although the interaction between the two bubbles becomes zero in this case. But, in the perpendicular situation shown in Fig. 10(b), the kinetic energy of the fluid increases as $H$ decrease or $\lambda_{1}$ increases. And, for the case of two identical bubbles, it has a maximum of $23 \%$ more than $E_{k \mathrm{o}}$ with the two identical bubbles touching each other.

\section{CONCLUSIONS}

This paper presents an explicit expression for the planar thermocapillary migration velocities of two bubbles in microgravity environment and investigates the interaction between them without considering, for convenience, bubble de-

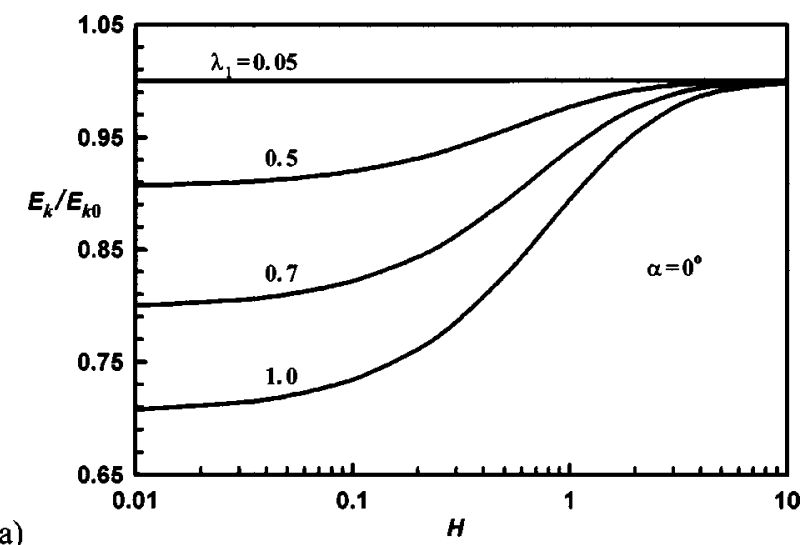

(a)

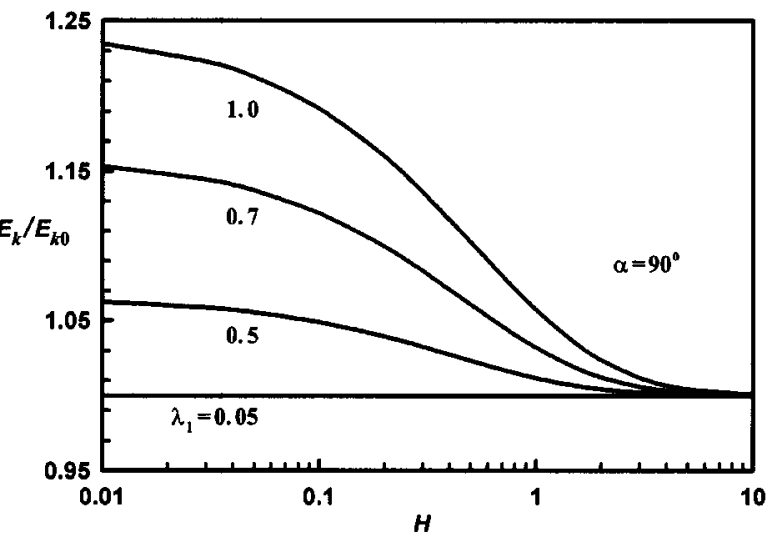

FIG. 10. Kinetic energy of the fluid transformed from heat energy versus the dimensionless gap $H$. (a) shows the case of $\alpha=0^{\circ}$, and (b) gives the situation with $\alpha=90^{\circ}$.

formation and van der Waals forces, although they have influence on the bubble interaction at a close range.

The results indicate that the interaction between two bubbles has significant influence on the migration of the smaller bubble. This effect mainly is concentrated in an interaction region, which is about one diameter apart from the larger bubble. If a smaller bubble intrudes into the zone, its velocity changes greatly due to the interaction. There is still a corresponding conclusion for the planar migration of two identical bubbles, that is, each of two equal-sized bubbles moves at the velocity that it would have as if it were isolated, independent of the dimensionless gap $H$ and orientation angle $\alpha$. When a larger bubble migrates in the proximity of other smaller bubbles, a larger bubble would move even beyond its typical speed in the isolated case. In addition, in the course of thermocapillary migration, when a tiny bubble gets to touch another larger bubble without coalescence, it will slide on the surface of the larger one down until reaching the stable position at $\alpha=180^{\circ}$.

Moreover, there are three typical dynamical behaviors for a smaller bubble migration near a larger one, a folium pattern, a cissoid pattern, and a witch pattern. They are determined by both an initial array of two bubbles and the size ratio. As the size ratio $\lambda_{1}$ is greater than the critical value $\lambda_{1 c r}$, the smaller bubble demonstrates only a witch-pattern behavior. These results indicate the initial configuration of 
two bubbles has a decisive influence on the subsequent migration of the smaller bubble.

The transformation of the thermal energy into the kinetic one on the surfaces of two bubbles varies with the variation of the interaction between the two bubbles, so such energy transformation differs in diverse configurations. In the axisymmetric case, the energy transformation diminishes with the decrease in the separation distance or increase in the size ratio $\lambda_{1}$ of the two bubbles, whereas it intensifies as the separation distance decreases or the size ratio $\lambda_{1}$ increases in the perpendicular case, and it gets the two extremes as $\lambda_{1}$ $=1$ and $H \rightarrow 0$.

\section{ACKNOWLEDGMENT}

This work was supported in part by the National Natural Science Foundation under Grant No. 19789201.

\section{APPENDIX: DERIVATION OF VELOCITY FIELDS AND TEMPERATURE DISTRIBUTIONS FOR A TWO-BUBBLE SYSTEM IN RELATION TO VARIOUS INITIAL DISTURBANCES}

In this appendix, a successive reflection procedure corresponding to that given by Sun and Chwang ${ }^{18,19}$ is used to deal with the Stokes equation and energy equation, and the fundament solutions will be discussed separately according to their mathematical structure.

\section{Transformations of harmonics and biharmonics between two coordinate systems}

In order to adopt the method of successive reflections in the following discussion on the interaction between two bubbles, we have to look for the transformations of harmonics and biharmonics between two coordinate systems. A pair of transformations of harmonics between $\left(r_{1}, \theta_{1}, \varphi\right)$ and $\left(r_{2}, \theta_{2}, \varphi\right)$ (see Fig. 1) was given by Hobson ${ }^{20}$ as below

$$
\frac{\mathrm{P}_{n}^{m} \cos m \varphi}{r_{1}^{n+1}}=(-1)^{n-m} \sum_{k=m}^{\infty} C_{m n k} r_{2}^{k} \mathrm{P}_{k}^{m} \cos m \varphi,
$$

and

$$
\frac{\mathrm{P}_{n}^{m} \cos m \varphi}{r_{2}^{n+1}}=\sum_{k=m}^{\infty}(-1)^{k-m} C_{m n k} r_{1}^{k} P_{k}^{m} \cos m \varphi,
$$

where $C_{m n k}=\left(\begin{array}{c}n+k \\ n-m\end{array}\right) / s^{n+k+1}(n \geqslant m)$. Here, $P_{n}^{m}$ and $\mathrm{P}_{n}^{m}$ denote $P_{n}^{m}\left(\cos \theta_{1}\right)$ and $P_{n}^{m}\left(\cos \theta_{2}\right)$, respectively. And, after some manipulation, biharmonic, $r_{1}^{2} \nabla\left(P_{n}^{m} \cos m \varphi / r_{1}^{n+1}\right)$, may be expanded in the $\left(r_{2}, \theta_{2}, \varphi\right)$ coordinates as

$$
\begin{aligned}
r_{1}^{2} \nabla\left(\frac{P_{n}^{m} \cos m \varphi}{r_{1}^{n+1}}\right) \\
=(-1)^{n-m} \sum_{k=m}^{\infty} C_{m n k}\left\{r_{2}^{2} \nabla\left(r_{2}^{k} \mathrm{P}_{k}^{m} \cos m \varphi\right)\right. \\
\quad-\frac{2(n+k+1)}{2 k+3} \nabla\left(r_{2}^{k+2} \mathrm{P}_{k}^{m} \cos m \varphi\right) \\
+s^{2}\left(1-\frac{2\left(k^{2}-m^{2}\right)}{(2 k-1)(n+k)}\right) \\
\left.\quad \times \nabla\left(r_{2}^{k} \mathrm{P}_{k}^{m} \cos m \varphi\right)+2 s \mathbf{e}_{3}^{\prime} r_{2}^{k} \mathrm{P}_{k}^{m} \cos m \varphi\right\},
\end{aligned}
$$

with the radius of convergence being $r_{2}<s$ by the ratio test.

Similarly

$$
\begin{aligned}
r_{2}^{2} \nabla\left(\frac{P_{n}^{m} \cos m \varphi}{r_{2}^{n+1}}\right) \\
=\sum_{k=m}^{\infty}(-1)^{k-m} C_{m n k}\left\{r_{1}^{2} \nabla\left(r_{1}^{k} P_{k}^{m} \cos m \varphi\right)\right. \\
\quad-\frac{2(n+k+1)}{2 k+3} \nabla\left(r_{1}^{k+2} P_{k}^{m} \cos m \varphi\right) \\
\quad+s^{2}\left(1-\frac{2\left(k^{2}-m^{2}\right)}{(2 k-1)(n+k)}\right) \\
\left.\quad \times \nabla\left(r_{1}^{k} P_{k}^{m} \cos m \varphi\right)-2 s \mathbf{e}_{3}^{\prime} r_{1}^{k} P_{k}^{m} \cos m \varphi\right\} .
\end{aligned}
$$

The radius of convergence for the summation in Eq. (A2b) is $r_{1}<s$.

\section{Derivation of velocity fields and temperature distributions for a two-bubble system in relation to various initial disturbances}

Suppose that the initial disturbance velocity created by bubble 1 takes the form of Eq. (5). It consists of harmonics and biharmonics. For convenience, let us consider an initial disturbance given by $\mathbf{h}_{m 0}^{\prime}$ is a harmonic in form and which, in the $\left(r_{1}, \theta_{1}, \varphi\right)$ coordinates, may be written as

$$
\mathbf{h}_{m 0}^{\prime[1]}=\nabla\left(\frac{P_{1}^{m} \cos m \varphi}{r_{1}^{2}}\right), \quad m=0 \text { or } 1 .
$$

Here, superscripts [1] and [2] mean that physical variables are expressed in the $\left(r_{1}, \theta_{1}, \varphi\right)$ and $\left(r_{2}, \theta_{2}, \varphi\right)$, respectively.

When a second bubble is released motionless in the flow at $o_{2}$, around bubble $2, \mathbf{h}_{m 0}^{\prime}$ can be expressed by using the transformation in (A1a) as

$$
\begin{aligned}
\mathbf{h}_{m 0}^{\prime[2]} & =(-1)^{1-m} \sum_{k=m}^{\infty} C_{m 1 k} \nabla\left(r_{2}^{k} \mathrm{P}_{k}^{m} \cos m \varphi\right) \\
& =\sum_{k=m}^{\infty} \lambda_{1 m k}^{(1)} \nabla\left(r_{2}^{k} \mathrm{P}_{k}^{m} \cos m \varphi\right),
\end{aligned}
$$


where $\lambda_{1 m k}^{(1)}=(-1)^{1-m} C_{m 1 k}$. From the impenetrable boundary condition on the surface of bubble 2 , we should put a corresponding correction $\mathbf{h}_{m 1}^{\prime}$

$$
\mathbf{h}_{m 1}^{\prime[2]}=\sum_{k=m}^{\infty} \frac{k}{k+1} \lambda_{1 m k}^{(1)} R_{2}^{2 k+1} \nabla\left(\frac{\mathrm{P}_{k}^{m} \cos m \varphi}{r_{2}^{k+1}}\right),
$$

into the flow field. Based on transformation (A1b), $\mathbf{h}_{m 1}^{\prime}$ in the $\left(r_{1}, \theta_{1}, \varphi\right)$ coordinates takes the form

$$
\mathbf{h}_{m 1}^{\prime[1]}=\sum_{k=m}^{\infty} \lambda_{1 m k}^{(2)} \nabla\left(r_{1}^{k} P_{k}^{m} \cos m \varphi\right),
$$

where $\lambda_{1 m k}^{(2)}=(-1)^{k-m} \sum_{l=m}^{\infty} l R_{2}^{2 l+1} \lambda_{1 m l}^{(1)} C_{m l k} /(l+1)$. But, the added correction makes an extra contribution to the flow field and breaks the impenetrable condition on bubble 1, and we should then introduce another compensating correction to counteract the violation. Continuing the same procedure in succession leads to an infinite sequence of reflection corrections. And, after some algebraic treatment, the velocity field outside the two bubbles in relation to the disturbance $\mathbf{h}_{m 0}^{\prime}$ finally becomes

$$
\begin{aligned}
\mathbf{h}_{\mathbf{m}}^{\prime}= & \nabla\left(\frac{P_{1}^{m} \cos m \varphi}{r_{1}^{2}}\right) \\
& +\sum_{k=m}^{\infty} \frac{k R_{2}^{2 k+1}}{k+1} \nabla\left(\frac{\mathrm{P}_{k}^{m} \cos m \varphi}{r_{2}^{k+1}}\right) \sum_{i=0}^{\infty} \lambda_{1 m k}^{(2 i+1)} \\
& +\sum_{k=m}^{\infty} \frac{k R_{1}^{2 k+1}}{k+1} \nabla\left(\frac{P_{k}^{m} \cos m \varphi}{r_{1}^{k+1}}\right) \sum_{i=0}^{\infty} \lambda_{1 m k}^{(2 i)}, \quad m=0 \text { or } 1,
\end{aligned}
$$

where iterative expressions of $\lambda_{1 m k}^{(i)}$ are

$$
\begin{aligned}
& \lambda_{1 m k}^{(0)}=0, \quad \lambda_{1 m k}^{(1)}=(-1)^{1-m} C_{m 1 k}, \\
& \lambda_{1 m k}^{(2 i)}=(-1)^{k-m} \sum_{l=m}^{\infty} \frac{l R_{2}^{2 l+1} \lambda_{1 m l}^{(2 i-1)} C_{m l k}}{l+1}, \\
& \lambda_{1 m k}^{(2 i+1)}=\sum_{l=m}^{\infty} \frac{(-1)^{l-m} l R_{1}^{2 l+1} \lambda_{1 m l}^{(2 i)} C_{m l k}}{l+1} .
\end{aligned}
$$

Similarly, let us next consider another initial disturbance, $\mathbf{h}_{m 0}^{\prime \prime}$, which appears in biharmonic form

$$
\mathbf{h}_{m 0}^{\prime \prime[1]}=r_{1}^{2} \nabla\left(\frac{P_{1}^{m} \cos m \varphi}{r_{1}^{2}}\right), \quad m=0 \text { or } 1 .
$$

To satisfy the impenetrable conditions on two bubble surfaces, we choose harmonics as subsequent reflection corrections for simplicity since a harmonic is a kind of biharmonics in nature. From transformations (A2a), (A1a), and (A1b), the velocity field outside the two-bubble system in relation to the disturbance $\mathbf{h}_{m 0}^{\prime \prime}$ is

$$
\begin{aligned}
\mathbf{h}_{\mathbf{m}}^{\prime \prime}= & r_{1}^{2} \nabla\left(\frac{P_{1}^{m} \cos m \varphi}{r_{1}^{2}}\right) \\
& +\sum_{k=m}^{\infty} \frac{k R_{2}^{2 k+1}}{k+1} \nabla\left(\frac{\mathrm{P}_{k}^{m} \cos m \varphi}{r_{2}^{k+1}}\right) \sum_{i=0}^{\infty} \mu_{1 m k}^{(2 i+1)} \\
& +\mathbf{e}_{3}^{\prime} \sum_{k=m}^{\infty} \frac{R_{2}^{2 k+1} \mathrm{P}_{k}^{m} \cos m \varphi}{r_{2}^{k+1}} \sum_{i=0}^{\infty} \nu_{1 m k}^{(2 i+1)} \\
& +\sum_{k=m}^{\infty} \frac{k R_{1}^{2 k+1}}{k+1} \nabla\left(\frac{P_{k}^{m} \cos m \varphi}{r_{1}^{k+1}}\right) \sum_{i=0}^{\infty} \mu_{1 m k}^{(2 i)} \\
& +\mathbf{e}_{3}^{\prime} \sum_{k=m}^{\infty} \frac{R_{1}^{2 k+1} P_{k}^{m} \cos m \varphi}{r_{1}^{k+1}} \sum_{i=0}^{\infty} \nu_{1 m k}^{(2 i)}, \quad m=0 \text { or } 1,
\end{aligned}
$$

with a set of iterative formulas

$$
\begin{aligned}
& \mu_{1 m k}^{(0)}=\nu_{1 m k}^{(0)}=0, \\
& \mu_{1 m k}^{(1)}=(-1)^{1-m} C_{m 1 k}\left\{s^{2}\left(1-\frac{2\left(k^{2}-m^{2}\right)}{(2 k-1)(k+1)}\right)\right. \\
& \left.-\frac{(5 k+8) R_{2}^{2}}{k(2 k+3)}\right\} \text {, } \\
& \nu_{1 m k}^{(1)}=(-1)^{m} 2 s C_{m 1 k}, \\
& \mu_{1 m k}^{(2 i)}=(-1)^{k-m} \sum_{l=m}^{\infty} \frac{l R_{2}^{2 l+1} \mu_{1 m l}^{(2 i-1)} C_{m l k}}{l+1}, \\
& \nu_{1 m k}^{(2 i)}=(-1)^{k-m+1} \sum_{l=m}^{\infty} R_{2}^{2 l+1} \nu_{1 m l}^{(2 i-1)} C_{m l k}, \\
& \mu_{1 m k}^{(2 i+1)}=\sum_{l=m}^{\infty} \frac{(-1)^{l-m} l R_{1}^{2 l+1} \mu_{1 m l}^{(2 i)} C_{m l k}}{l+1}, \\
& \nu_{1 m k}^{(2 i+1)}=\sum_{l=m}^{\infty}(-1)^{l-m+1} R_{1}^{2 l+1} \nu_{1 m l}^{(2 i)} C_{m l k} .
\end{aligned}
$$

And, as the third initial disturbance, $\mathbf{h}_{m 0}^{\prime \prime \prime}$ is a harmonic below

$$
\mathbf{h}_{m 0}^{\prime \prime \prime}[1]=\frac{\mathbf{e}_{3-m}^{\prime}}{r_{1}}, \quad m=0 \text { or } 1 .
$$

Based on transformations (A1a) and (A1b), the velocity field outside the two bubbles in relation to $\mathbf{h}_{m 0}^{\prime \prime \prime}$ is as follows:

$$
\begin{aligned}
\mathbf{h}_{\mathbf{m}}^{\prime \prime \prime}= & \mathbf{e}_{3-m}^{\prime}\left\{\frac{1}{r_{1}}+\sum_{k=0}^{\infty} \frac{R_{2}^{2 k+1} \mathrm{P}_{k}}{r_{2}^{k+1}} \sum_{i=0}^{\infty} \gamma_{1 k}^{(2 i+1)}\right. \\
& \left.+\sum_{k=0}^{\infty} \frac{R_{1}^{2 k+1} P_{k}}{r_{1}^{k+1}} \sum_{i=0}^{\infty} \gamma_{1 k}^{(2 i)}\right\}, \quad m=0 \text { or } 1,
\end{aligned}
$$

where 


$$
\begin{aligned}
& \gamma_{1 k}^{(0)}=0, \quad \gamma_{1 k}^{(1)}=-C_{00 k}, \\
& \gamma_{1 k}^{(2 i)}=(-1)^{k+1} \sum_{l=0}^{\infty} R_{2}^{2 l+1} \gamma_{1 l}^{(2 i-1)} C_{0 l k}, \\
& \gamma_{1 k}^{(2 i+1)}=\sum_{l=0}^{\infty}(-1)^{l+1} R_{1}^{2 l+1} \gamma_{1 l}^{(2 i)} C_{0 l k} .
\end{aligned}
$$

Consider next the contribution to the velocity field just from the initial disturbances generated by bubble 2 when bubble 1 is inserted at a standstill nearby. In the same way, the velocity field exterior to the whole system in relation to the initial disturbance $\mathbf{H}_{m 0}^{\prime}$, which in the $\left(r_{2}, \theta_{2}, \varphi\right)$ coordinates is written as

$$
\mathbf{H}_{m 0}^{\prime[2]}=\nabla\left(\frac{\mathrm{P}_{1}^{m} \cos m \varphi}{r_{2}^{2}}\right), \quad m=0 \text { or } 1,
$$

is expressed as

$$
\begin{aligned}
\mathbf{H}_{m}^{\prime}= & \nabla\left(\frac{\mathrm{P}_{1}^{m} \cos m \varphi}{r_{2}^{2}}\right) \\
& +\sum_{k=m}^{\infty} \frac{k R_{1}^{2 k+1}}{k+1} \nabla\left(\frac{P_{k}^{m} \cos m \varphi}{r_{1}^{k+1}}\right) \sum_{i=0}^{\infty} \lambda_{2 m k}^{(2 i+1)} \\
& +\sum_{k=m}^{\infty} \frac{k R_{2}^{2 k+1}}{k+1} \nabla\left(\frac{\mathrm{P}_{k}^{m} \cos m \varphi}{r_{2}^{k+1}}\right) \sum_{i=0}^{\infty} \lambda_{2 m k}^{(2 i)}, \quad m=0 \text { or } 1,
\end{aligned}
$$

where

$$
\begin{aligned}
& \lambda_{2 m k}^{(0)}=0, \quad \lambda_{2 m k}^{(1)}=(-1)^{k-m} C_{m 1 k}, \\
& \lambda_{2 m k}^{(2 i)}=\sum_{l=m}^{\infty} \frac{(-1)^{l-m} l R_{1}^{2 l+1} \lambda_{2 m l}^{(2 i-1)} C_{m l k}}{l+1}, \\
& \lambda_{2 m k}^{(2 i+1)}=(-1)^{k-m} \sum_{l=m}^{\infty} \frac{l R_{2}^{2 l+1} \lambda_{2 m l}^{(2 i)} C_{m l k}}{l+1} .
\end{aligned}
$$

The exterior velocity field in relation to

$$
\mathbf{H}_{m 0}^{\prime \prime[2]}=r_{2}^{2} \nabla\left(\frac{\mathrm{P}_{1}^{m} \cos m \varphi}{r_{2}^{2}}\right), \quad m=0 \text { or } 1,
$$

becomes

$$
\begin{aligned}
\mathbf{H}_{m}^{\prime \prime}= & r_{2}^{2} \nabla\left(\frac{\mathrm{P}_{1}^{m} \cos m \varphi}{r_{2}^{2}}\right) \\
& +\sum_{k=m}^{\infty} \frac{k R_{1}^{2 k+1}}{k+1} \nabla\left(\frac{P_{k}^{m} \cos m \varphi}{r_{1}^{k+1}}\right) \sum_{i=0}^{\infty} \mu_{2 m k}^{(2 i+1)} \\
& +\mathbf{e}_{3}^{\prime} \sum_{k=m}^{\infty} \frac{R_{1}^{2 k+1} P_{k}^{m} \cos m \varphi}{r_{1}^{k+1}} \sum_{i=0}^{\infty} \nu_{2 m k}^{(2 i+1)} \\
& +\sum_{k=m}^{\infty} \frac{k R_{2}^{2 k+1}}{k+1} \nabla\left(\frac{\mathrm{P}_{k}^{m} \cos m \varphi}{r_{2}^{k+1}}\right) \sum_{i=0}^{\infty} \mu_{2 m k}^{(2 i)} \\
& +\mathbf{e}_{3}^{\prime} \sum_{k=m}^{\infty} \frac{R_{2}^{2 k+1} \mathrm{P}_{k}^{m} \cos m \varphi}{r_{2}^{k+1}} \sum_{i=0}^{\infty} \nu_{2 m k}^{(2 i)}, \quad m=0 \text { or } 1,
\end{aligned}
$$

where

$$
\begin{aligned}
& \mu_{2 m k}^{(0)}= \nu_{2 m k}^{(0)}=0, \\
& \mu_{2 m k}^{(1)}=(-1)^{k-m} C_{m 1 k}\left\{s^{2}\left(1-\frac{2\left(k^{2}-m^{2}\right)}{(2 k-1)(k+1)}\right)\right. \\
&\left.-\frac{(5 k+8) R_{1}^{2}}{k(2 k+3)}\right\}, \\
& \nu_{2 m k}^{(1)}=(-1)^{k-m} 2 s C_{m 1 k}, \\
& \mu_{2 m k}^{(2 i)}= \sum_{l=m}^{\infty} \frac{(-1)^{l-m} l R_{1}^{2 l+1} \mu_{2 m l}^{(2 i-1)} C_{m l k}}{l+1}, \\
& \nu_{2 m k}^{(2 i)}= \sum_{l=m}^{\infty}(-1)^{l-m+1} R_{1}^{2 l+1} \nu_{2 m l}^{(2 i-1)} C_{m l k}, \\
& \mu_{2 m k}^{(2 i+1)}=(-1)^{k-m} \sum_{l=m}^{\infty} \frac{l R_{2}^{2 l+1} \mu_{2 m l}^{(2 i)} C_{m l k}}{l+1}, \\
& \nu_{2 m k}^{(2 i+1)}=(-1)^{k-m+1} \sum_{l=m}^{\infty} R_{2}^{2 l+1} \nu_{2 m l}^{(2 i)} C_{m l k} .
\end{aligned}
$$

And, such outside velocity field in relation to

$$
\mathbf{H}_{m 0}^{\prime \prime \prime[2]}=\frac{\mathbf{e}_{3-m}^{\prime}}{r_{2}}, \quad m=0 \text { or } 1,
$$

is

$$
\begin{aligned}
\mathbf{H}_{m}^{\prime \prime \prime}= & \mathbf{e}_{3-m}^{\prime}\left\{\frac{1}{r_{2}}+\sum_{k=0}^{\infty} \frac{R_{1}^{2 k+1} P_{k}}{r_{1}^{k+1}} \sum_{i=0}^{\infty} \gamma_{2 k}^{(2 i+1)}\right. \\
& \left.+\sum_{k=0}^{\infty} \frac{R_{2}^{2 k+1} \mathrm{P}_{k}}{r_{2}^{k+1}} \sum_{i=0}^{\infty} \gamma_{2 k}^{(2 i)}\right\}, \quad m=0 \text { or } 1,
\end{aligned}
$$

where

$$
\begin{aligned}
& \gamma_{2 k}^{(0)}=0, \quad \gamma_{2 k}^{(1)}=(-1)^{k+1} C_{00 k}, \\
& \gamma_{2 k}^{(2 i)}=\sum_{l=0}^{\infty}(-1)^{l+1} R_{1}^{2 l+1} \gamma_{2 l}^{(2 i-1)} C_{0 l k}, \\
& \gamma_{2 k}^{(2 i+1)}=(-1)^{k+1} \sum_{l=0}^{\infty} R_{2}^{2 l+1} \gamma_{2 l}^{(2 i)} C_{0 l k} .
\end{aligned}
$$

Moreover, for an initial disturbance in temperature caused by bubble 1

$$
t_{m 0}^{\prime[1]}=\frac{P_{1}^{m} \cos m \varphi}{r_{1}^{2}}, \quad m=0 \text { or } 1,
$$

the temperature distribution exterior to the two bubbles becomes 


$$
\begin{aligned}
t_{m}^{\prime}= & \frac{P_{1}^{m} \cos m \varphi}{r_{1}^{2}}+\sum_{k=m}^{\infty} \frac{k R_{2}^{2 k+1} \mathrm{P}_{k}^{m} \cos m \varphi}{(k+1) r_{2}^{k+1}} \sum_{i=0}^{\infty} \phi_{1 m k}^{(2 i+1)} \\
& +\sum_{k=m}^{\infty} \frac{k R_{1}^{2 k+1} P_{k}^{m} \cos m \varphi}{(k+1) r_{1}^{k+1}} \sum_{i=0}^{\infty} \phi_{1 m k}^{(2 i)}, \quad m=0 \text { or } 1,
\end{aligned}
$$

where

$$
\begin{aligned}
& \phi_{1 m k}^{(0)}=0, \quad \phi_{1 m k}^{(1)}=(-1)^{1-m} C_{m 1 k}, \\
& \phi_{1 m k}^{(2 i)}=(-1)^{k-m} \sum_{l=m}^{\infty} \frac{l R_{2}^{2 l+1} \phi_{1 m l}^{(2 i-1)} C_{m l k}}{l+1}, \\
& \phi_{1 m k}^{(2 i+1)}=\sum_{l=m}^{\infty} \frac{(-1)^{l-m} l R_{1}^{2 l+1} \phi_{1 m l}^{(2 i)} C_{m l k}}{l+1},
\end{aligned}
$$

whereas for the other initial disturbance in temperature aroused by bubble 2

$$
\tau_{m 0}^{\prime[2]}=\frac{\mathrm{P}_{1}^{m} \cos m \varphi}{r_{2}^{2}}, \quad m=0 \text { or } 1,
$$

the temperature distribution outside the whole system is

$$
\begin{aligned}
\tau_{m}^{\prime}= & \frac{\mathrm{P}_{1}^{m} \cos m \varphi}{r_{2}^{2}}+\sum_{k=m}^{\infty} \frac{k R_{1}^{2 k+1} P_{k}^{m} \cos m \varphi}{(k+1) r_{1}^{k+1}} \sum_{i=0}^{\infty} \phi_{2 m k}^{(2 i+1)} \\
& +\sum_{k=m}^{\infty} \frac{k R_{2}^{2 k+1} \mathrm{P}_{k}^{m} \cos m \varphi}{(k+1) r_{2}^{k+1}} \sum_{i=0}^{\infty} \phi_{2 m k}^{(2 i)}, \quad m=0 \text { or } 1,
\end{aligned}
$$

where

$$
\begin{aligned}
& \phi_{2 m k}^{(0)}=0, \quad \phi_{2 m k}^{(1)}=(-1)^{k-m} C_{m 1 k}, \\
& \phi_{2 m k}^{(2 i)}=\sum_{l=m}^{\infty} \frac{(-1)^{l-m} l R_{1}^{2 l+1} \phi_{2 m l}^{(2 i-1)} C_{m l k}}{l+1}, \\
& \phi_{2 m k}^{(2 i+1)}=(-1)^{k-m} \sum_{l=m}^{\infty} \frac{l R_{2}^{2 l+1} \phi_{2 m l}^{(2 i)} C_{m l k}}{l+1} .
\end{aligned}
$$

Every recurrence formula above holds for all positive integers $k$ and $i$.

${ }^{1}$ N. O. Young, J. S. Goldstein, and M. J. Block, "The motion of bubbles in a vertical temperature gradient," J. Fluid Mech. 6, 350 (1959).

${ }^{2}$ G. Wozniak, J. Siekmann, and J. Srulijes, "Thermocapillary bubble and drop dynamics under reduced gravity-survey and prospects," Z. Flugwiss. Weltraumforsch. 12, 137 (1988).

${ }^{3}$ R. S. Subramanian, "The motion of bubbles and drops in reduced gravity," in Transport Processes in Bubbles, Drops and Particles, edited by R. P. Chhabra and D. Dekee (Hemisphere, New York, 1992), pp. 1-42.

${ }^{4}$ M. Meyyappan, W. R. Wilcox, and R. S. Subramanian, "The slow axisymmetric motion of two bubbles in a thermal gradient," J. Colloid Interface Sci. 94, 243 (1983).

${ }^{5} \mathrm{~F}$. Feuillebois, "Thermocapillary migration of two equal bubbles parallel to their line of centers," J. Colloid Interface Sci. 131, 267 (1989).

${ }^{6} \mathrm{M}$. Meyyappan and R. S. Subramanian, "The thermocapillary motion of two bubbles oriented arbitrarily relative to a thermal gradient," J. Colloid Interface Sci. 97, 291 (1984).

${ }^{7}$ S. Kim and S. J. Karrila, Microhydrodynamics: Principles and Selected Applications (Butterworths-Heinemann, London, 1991).

${ }^{8}$ J. L. Anderson, "Droplet interactions in thermocapillary motion," Int. J. Multiphase Flow 11, 813 (1985).

${ }^{9}$ A. Acrivos, D. J. Jeffrey, and D. A. Saville, "Particle migration in suspensions by thermocapillary or electrophoretic motion," J. Fluid Mech. 212, 95 (1990).

${ }^{10}$ H. J. Keh and L. S. Chen, "Droplet interactions in axisymmetric thermocapillary motion," J. Colloid Interface Sci. 151, 1 (1992).

${ }^{11} \mathrm{H}$. Wei and R. S. Subramanian, "Thermocapillary migration of a small chain of bubbles," Phys. Fluids A 5, 1583 (1993).

${ }^{12} \mathrm{~J}$. V. Satrape, "Interactions and collisions of bubbles in thermocapillary motion," Phys. Fluids A 4, 1883 (1992).

${ }^{13}$ Y. Wang, R. Mauri, and A. Acrivos, "Thermocapillary migration of a bidisperse suspension of bubbles," J. Fluid Mech. 261, 47 (1994).

${ }^{14}$ R. Sun and W. R. Hu, "The thermocapillary migrations of two bubbles in microgravity environment," J. Colloid Interface Sci. 255, 375 (2002).

${ }^{15}$ R. S. Subramanian, "Slow migration of a gas bubble in a thermal gradient," AIChE J. 27, 646 (1981).

${ }^{16} \mathrm{H}$. Lamb, Hydrodynamics, 6th ed. (Cambridge University Press, Cambridge, 1932).

${ }^{17}$ W. Cheney and D. Kincaid, Numerical Mathematics and Computing (Brooks/Cole, Pacific Grove, CA, 1999).

${ }^{18}$ R. Sun and A. T. Chwang, "Hydrodynamic interaction between two cylinders with rotation,” J. Phys. Soc. Jpn. 70, 91 (2001).

${ }^{19}$ R. Sun and A. T. Chwang, "Hydrodynamic interaction between a slightly distorted sphere and a fixed sphere," Theor. Comput. Fluid Dyn. 15, 11 (2001).

${ }^{20}$ E. W. Hobson, The Theory of Spherical and Ellipsoidal Harmonics (Chelsea, New York, 1955). 